\title{
Influenza Illness in Pregnant Indian Women: A Cross-Sectional Study
}

\author{
Parvaiz A. Koul, ${ }^{1}$ Nargis K. Bali, ${ }^{2}$ Hyder Mir, ${ }^{1}$ Farhat Jabeen, ${ }^{3}$ and Abida Ahmad ${ }^{4}$ \\ ${ }^{1}$ Department of Internal \& Pulmonary Medicine, Sher-I-Kashmir Institute of Medical Sciences, Soura, Srinagar 190011, India \\ ${ }^{2}$ Department of Clinical Microbiology, Sher-I-Kashmir Institute of Medical Sciences, Soura, Srinagar 190011, India \\ ${ }^{3}$ Department of Obstetrics and Gynecology, Lalla Ded Hospital for Women, Government Medical College, Srinagar 190010, India \\ ${ }^{4}$ Department of Obstetrics and Gynecology, Sher-I-Kashmir Institute of Medical Sciences, Soura, Srinagar 190011, India
}

Correspondence should be addressed to Parvaiz A. Koul; parvaizk@gmail.com

Received 9 October 2015; Revised 20 December 2015; Accepted 29 December 2015

Academic Editor: Lu-Yu Hwang

Copyright (c) 2016 Parvaiz A. Koul et al. This is an open access article distributed under the Creative Commons Attribution License, which permits unrestricted use, distribution, and reproduction in any medium, provided the original work is properly cited.

\begin{abstract}
Data about burden of influenza in pregnancy in India are scant. In order to assess the contribution of influenza to acute respiratory illness (ARI) in pregnancy, 266 north Indian pregnant females with febrile ARI were studied from December 2014 to May 2015. Twin nasopharyngeal/oropharyngeal swabs were obtained and tested for influenza viruses by RT-PCR. Fifty (18.8\%) patients tested positive for influenza (A/H1N1pdm09 in 41, A/H3N2 in 8, and influenza B Yamagata in 1). Rigors, headache, and a family history of ARI were significantly more frequent in influenza positive patients. Oseltamivir and supportive therapy were administered to all confirmed cases. Nine influenza positive cases needed hospitalization for their respiratory illness, and 5 developed respiratory failure. Of these, 4 (3 in third trimester) succumbed to their illness. We conclude that influenza viruses are a cause of significant morbidity and mortality among pregnant females with ARI in north India. As such, appropriate preventive strategies of influenza vaccination and early initiation of antiviral therapy during illness are stressed.
\end{abstract}

\section{Introduction}

Influenza during pregnancy has been associated with considerable morbidity and mortality. Pregnant women were observed to be at high risk of complications such as pneumonia and death during the influenza pandemics of 1918, 1957, and 2009. In the 1918 pandemic, Harris described an overall mortality of $27 \%$ among pregnant females who developed influenza-associated pneumonia which exceeded $50 \%$ in the third trimester of pregnancy [1]. In the 1957 pandemic, there was increased mortality due to influenza complicating pregnancy as compared to nonpregnant females [2]. Pregnant women accounted for about $6 \%$ of influenza related hospitalizations, ICU admissions, and deaths in the 2009 influenza pandemic, even though they constitute only $1 \%$ of the US population at any point in time $[3,4]$. In the 18-29 years age group, pregnancy accounted for up to $29 \%$ of influenza-associated hospitalizations and $16 \%$ of deaths [5-7]. About $50 \%$ of the pregnancy-associated deaths in the April-September $2009 \mathrm{H} 1 \mathrm{~N} 1$ pandemic period were observed in the third trimester whereas $36 \%$ occurred during the second trimester [8]. In a recent review of 100 studies published between 1961 and 2015, investigators reported that, compared to the general population, pregnant women are more often hospitalized and admitted to an intensive care unit due to influenza virus infection [9]. During May-June 2009 , pregnant women were 7.2 times more likely to be hospitalized and 4.3 times more likely to be admitted to an ICU than nonpregnant women [10]. Coexiting conditions such as asthma or diabetes put pregnant women at 34 times greater risk of morbidity as compared to nonpregnant control subjects with similar high-risk conditions [11].

Influenza has a significant impact on the mother as well as the fetus. Infection during pregnancy has been associated with an approximately fivefold increase in perinatal mortality, including miscarriages, stillbirths, and early neonatal diseases and death [11, 12]. A 3-fold increased risk of premature 
and complicated birth was observed in pregnant women hospitalized with A/H1N1pdm09 [13].

There are limited data describing the burden of influenza in pregnancy in India. Previously, Gunasekaran et al. found that a higher proportion of pregnant women were positive for both seasonal influenza (11.1\% pregnant women versus $1.4 \%$ nonpregnant women) and influenza A/H1Nlpdm09 (21.4\% pregnant women versus $2.7 \%$ nonpregnant women) [14]. However, Pramanick et al. reported that, among all women presenting with ILI/SARI, influenza A (pH1N1) was positive in $25.3 \%$ of pregnant/puerperal women and $29.6 \%$ of nonpregnant women [15]. In spite of high rate of morbidity and mortality, uptake of influenza vaccination in pregnant females is very low [16]. Therefore, it is imperative to improve our understanding of the burden of influenza viruses to respiratory illness in pregnancy so that preventive measures such as vaccination can be rationally implemented.

\section{Material and Methods}

The study was conducted in 2 tertiary care referral centers for obstetrics cases of the Kashmir Valley in the north Indian state of Jammu \& Kashmir in a cross-sectional design. We have previously documented a temperate seasonality of influenza circulation with wintertime peaks in this region with a significant contribution of influenza towards causation of respiratory illness [17]. About 350 to 400 women seeking obstetric care are seen in the Lalla Ded (LD) Hospital for Women whereas about 150 women were seen in Sher-IKashmir Institute of Medical Sciences (SKIMS). These hospitals cater to the majority of the institutional obstetrics cases in the valley. The sample population comprised women $(\geq 18$ years) presenting in any stage of pregnancy to the obstetrics departments with symptoms of influenza-like illness (ILI) (defined as a sudden onset of fever $\geq 38^{\circ} \mathrm{C}$, with cough or sore throat in the absence of any other diagnosis and an onset within the past 10 days) or severe acute respiratory infection (SARI) (defined as a patient with ILI who required admission for the respiratory illness). Patients without fever were labeled as acute respiratory illness (ARI).

2.1. Sample Collection and Testing. The study started in December 2014 and continued until May 2015 when the enrollment target was achieved. Clinical history and examination of the study patients were recorded for all participants including any history of clustering (two or more cases that were related in time and space, e.g., in a home or workplace) for the entire study period. Parity and obstetric history were recorded along with any history of complications of the pregnancy. Combined throat and nasal swabs were collected in viral transport medium at the LD Hospital/SKIMS and transported immediately to the Influenza Laboratory at SKIMS. All samples were processed within 3-4 hours of collection. Samples were tested by real-time RT-PCR for influenza viruses A and B using the standard CDC protocol [18]. All influenza A positive samples were further subtyped using primers and probes for $\mathrm{A} / \mathrm{H} 1 \mathrm{~N} 1 \mathrm{pdm} 09$ and $\mathrm{A} / \mathrm{H} 3$. Influenza $\mathrm{B}$ viruses were further subtyped into B/Yamagata and $B /$ Victoria subtypes using specific primers.
A confirmed case of influenza was defined as a study participant with ILI/SARI/ARI with laboratory-confirmed influenza A or B detected by RT-PCR.

2.2. Analysis. Statistical analysis of the data was performed using SPSS statistical software version 11.0, IBM Corp., USA. The clinical features of influenza positive and influenza negative patients were compared. Data have been expressed as Mean $\pm \mathrm{SD}$. Categorical variables were compared using Fisher's exact/Chi-square test and continuous variables by employing Student's $t$-test. A $p$ value of $<0.05$ was considered significant.

2.3. Ethics. The study was approved by the Institute Ethics Committee of Sher-I-Kashmir Institute of Medical Sciences, Srinagar (protocol ID: RP 241/2014 of 2014).

\section{Results}

The 266 observed women were aged 18-39 years (median 27 years), 257 of which had ILI whereas 9 required hospitalization for their respiratory illness. While 141 patients were from the urban setting, 125 were from rural areas. The patients presented within 1-10 (median 4) days of the onset of symptoms that included fever, chills/rigors, cough, nasal discharge, sore throat, headache, and body aches. The parity status of the women was primigravida $(n=103)$, G2 $(n=71), \mathrm{G} 3(n=57), \mathrm{G} 4(n=22)$, G5 $(n=10)$, G6 $(n=2)$, and G7 $(n=1)$. The duration of pregnancy ranged from 5 weeks to 9 months (median 27.5 weeks); $144(66.7 \%)$ belonged to the third trimester, $94(43.5 \%)$ to the second trimester, and $28(12.9 \%)$ to the first trimester. Eleven patients had a history of recurrent pregnancy loss and 55 had a history of previous cesarean section. Comorbid illnesses included pregestational/gestational diabetes $(n=$ $11)$, gestational hypertension $(n=23)$, anaemia $(n=120)$, and hypothyroidism $(n=3)$. One of the patients had a twin pregnancy and one had a large-for-gestation baby. None had received influenza vaccination in the current pregnancy and or had been advised to receive it.

Fifty (18.8\%; age $20-35$ years; median, 20$)$ of the 266 observed patients tested positive for influenza viruses against the routine positivity of around $18 \%$ seen over a 5 -year period of surveillance [17]. Further subtyping of the isolates revealed that $41(82 \%)$ were positive for the A/H1N1pdm09, 8 (16\%) were positive for $\mathrm{A}(\mathrm{H} 3 \mathrm{~N} 2)$, and $1(2.0 \%)$ was positive for influenza B (Yamagata lineage). Of the 50 patients who tested positive for influenza viruses, $30(60 \%)$ were in the third trimester, 15 (30\%) were in the second, and $5(10 \%)$ were in the first. The relative positivity did not differ among the 3 trimesters, ranging from $15.9 \%$ to $20.8 \%(p>0.05)$. Various symptoms reported by influenza positive patients included fever with rigors $(n=48)$, cough $(n=47)$, body aches $(n=47)$, fatigue $(n=47)$, headache $(n=45)$, nasal discharge $(n=40)$, breathlessness $(n=40)$, sore throat $(n=41)$, expectoration $(n=30)$, vomiting $(n=8)$, and diarrhea $(n=5)$. Sixty-two of the observed patients $(44 \%$ of all influenza positive patients) had a history of an ARI/ILI in the family. Comorbidities in influenza positive patients 
TABLE 1: Clinical features of influenza positive and influenza negative patients.

\begin{tabular}{|c|c|c|c|}
\hline Clinical features & $\begin{array}{l}\text { Influenza negative } \\
\qquad \begin{array}{c}N=216 \\
N(\%)\end{array}\end{array}$ & $\begin{array}{l}\text { Influenza positive } \\
\qquad \begin{array}{c}N=50 \\
N(\%)\end{array}\end{array}$ & $p$ value \\
\hline Duration of symptoms (mean \pm SD range) in days; median & $4.25 \pm 1.98(1-10) ; 4$ & $3.74 \pm 2.03(1-10) ; 4$ & 0.10 \\
\hline Fever & $191(88.4)$ & $48(96)$ & 0.11 \\
\hline Rigors & $32(14.8)$ & $48(96)$ & $<0.0001$ \\
\hline Nasal discharge & $195(90.3)$ & $40(80)$ & 0.041 \\
\hline Ear discharge & $1(0.5)$ & $0(0)$ & \\
\hline Cough & $197(91.2)$ & $47(94)$ & 0.52 \\
\hline Sore throat & $177(81.9)$ & $41(82)$ & 0.98 \\
\hline Breathlessness & $182(84.3)$ & $40(80)$ & 0.46 \\
\hline Expectoration & $152(70.4)$ & $30(60)$ & 0.15 \\
\hline Headache & $132(61.1)$ & $45(90)$ & 0.0001 \\
\hline Body ache & $191(88.4)$ & $47(94)$ & 0.25 \\
\hline Fatigue & $192(88.9)$ & $47(94)$ & 0.28 \\
\hline Vomiting & $49(22.7)$ & $8(16)$ & 0.30 \\
\hline Diarrhea & $12(5.6)$ & $5(10)$ & 0.25 \\
\hline Seizures & $1(0.5)$ & $0(0)$ & \\
\hline ARTI/ILI in the family & $40(15.0)$ & $22(44)$ & $<0.0001$ \\
\hline
\end{tabular}

ARI = acute respiratory illness; ILI = influenza-like illness.

included pregestational/gestational hypertension $(n=6)$, pregestational/gestational diabetes $(n=3)$, and anemia $(n=26)$. The frequency of comorbidities like hypertension, diabetes, or anemia was not significantly different in influenza positive as compared to influenza negative participants $(p=$ $0.35,0.46$, and 0.41 , resp.). A comparative analysis of clinical presentations among influenza positive and influenza negative patients is given in Table 1. Rigors, headache, and nasal discharge were seen significantly more frequently in patients who tested influenza positive.

All patients who were influenza positive were administered oral Oseltamivir. Of the 50, nine required admission for severe respiratory illness (bilateral infiltrates with respiratory failure $(n=5)$ and lobar pneumonia $(n=4)$ (Figure 1$)$ ). Five of these required invasive mechanical ventilation for respiratory failure, broad spectrum antibiotics, and vasopressor agents. Four patients succumbed to multiorgan failure. Three of these were in the third trimester whereas one was in the second trimester. These patients had presented with respiratory symptoms of 2-5 days, were aged 21-29 years, and developed severe respiratory distress prior to presentation to the hospital. All were previously healthy and had no attendant comorbidities, and A/H1N1pdm09 was detected in all the 4. A premortem cesarean section in one patient led to the survival of the baby. Apart from these 4, all patients had an uncomplicated recovery.

\section{Discussion}

Our data suggest that influenza viruses are an important cause of respiratory illness in pregnant females with considerable morbidity and mortality. To the best of our knowledge, this study is the first one to document the burden of influenza

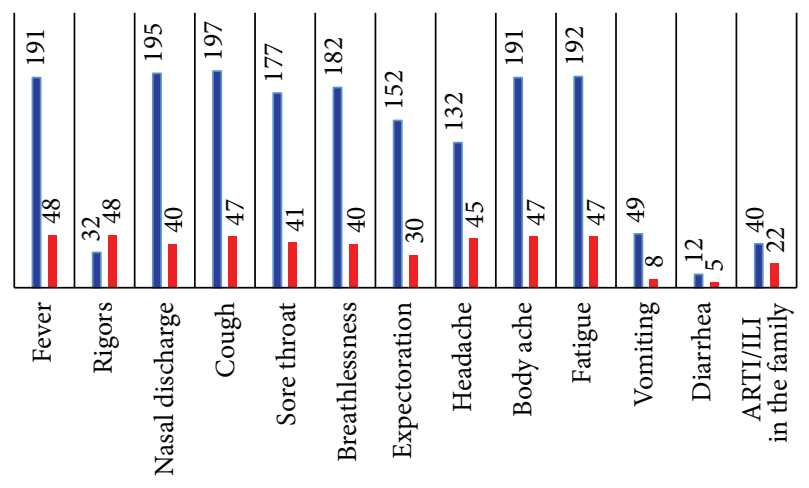

- Influenza negative $(n=216)$

- Influenza positive $(n=50)$

FIGURE 1: Graphical representation of the major symptoms at presentation.

in pregnant females in India employing active surveillance in pregnant women.

Influenza virus contributed to $18.8 \%$ of the acute respiratory infections in the observed pregnant females with $82 \%$ of the infections caused by the influenza A/H1N1pdm09, 16\% by $\mathrm{A} / \mathrm{H} 3 \mathrm{~N} 2$, and $2 \%$ by influenza $\mathrm{B}$ virus. The comparative prevalence of the various strains in the community surveillance included A/H1N1pdm09 (86\%), A/H3N2 (12\%), and B (Yamagata) 1\% (unpublished data), the strain distribution among the pregnant females being similar to the general community trends of circulation. While only two previous Indian reports exist of seasonal influenza viruses causing respiratory illness in pregnancy $[14,15]$, others have focused on pandemic A/H1N1pdm09 virus [19-23]. Predominance of $\mathrm{A} / \mathrm{H} 1 \mathrm{~N} 1 \mathrm{pdm} 09$ as the dominant virus causing respiratory illness in pregnant females was also reported earlier [14, 15]. 
However, we attribute this predominance of the strain to the general circulation of A/H1N1pdm09 during the season rather than any specific propensity of pregnant females to A/H1N1pdm09 as the strain was the major circulating strain at the time of the study (Koul PA. unpublished data).

The risk of hospitalization in pregnant women has been observed to be 18 -fold compared to nonpregnant women even during the interpandemic period, with risk being greatest among women in later stages of pregnancy [24-26]. The majority $(60 \%)$ of influenza cases in our study also occurred in the third trimester. Four of the 5 pregnant women who developed respiratory failure were in the third trimester of their pregnancy and 3 of them died. The higher risk of severe disease and ICU admission is consistent with earlier reports [2, 20-22,27], with high rates of complications such as pneumonia and renal failure. Pregnant women once infected seem to develop severe infection [28]. The case fatality rate in our study was $8 \%$, all being $\mathrm{A} / \mathrm{H} 1 \mathrm{~N} 1 \mathrm{pdm} 09$ positive. A/H1N1pdm09 influenza infection has been associated with higher mortality (25-70\%) among Indian pregnant women $[21,23,29]$ than that reported from other countries [30, 31]. Such high figures could be an overestimation as the total number of pregnant females in these studies is low. Different sampling methods and diverse study population could be other factors attributing to such high figures and there could be a contribution of relatively inadequate ICU facilities. However, Gunasekaran et al. reported a mortality of only 3.7\% [14]. While associated comorbidities like asthma and diabetes pose a higher risk of morbidity in pregnant females as compared to their nonpregnant controls [11, 24], none of our patients with adverse outcomes had a comorbid illness.

The mechanisms that increase the risk of serious complications from influenza in pregnancy are incompletely understood. A combination of cardiac, respiratory, hormonal, and immunological changes accompany pregnancy which impair responses to infection and increase the likelihood of serious complications that require admission to the hospital $[32,33]$. These changes include elevation of the diaphragm due to increased uterine size, increased intra-abdominal pressure, increased respiratory rate, reduced chest compliance, and high risk of aspiration as a consequence. Other physiological changes include reduced tidal volume and lung capacity and increased cardiac output and oxygen consumption. Decreases in adaptive immunity seen in later stages of pregnancy is consistent with the observed increase in the severity of certain infectious diseases during later pregnancy. Decreases in the numbers and function of CD4+, CD8+, and natural killer cells could affect antimicrobial responses and delay clearance of the infecting microorganism [34]. Additionally, while some cytokines are suppressed (e.g., IFN $\gamma$ and VEGF), others (e.g., the proinflammatory cytokines $\mathrm{TNF} \alpha$ and G-CSF) are increased throughout pregnancy. The cytokine changes result in interplay with resultant changes in Th1 and Th2 responses, NK cell function, and antigen presentation. When incubated with influenza viruses, peripheral blood mononuclear cells from third trimester pregnant women exhibit reduced antiviral gene expression and consequently higher replication of the viruses [35].
An important observation of the current study was that none of the pregnant females had received influenza vaccination despite being pregnant in the influenza season. Although routine vaccination of pregnant females against influenza with inactivated trivalent influenza vaccine is recommended [36], it has been only recently endorsed by the Federation of Obstetricians and Gynecologists of India (FOGSI) [37]. We have recently demonstrated a poor uptake of influenza vaccination among 1000 pregnant females in Kashmir where vaccinations were not adopted at all [16]. In absence of similar data from other parts of the country, these data more or less reflect the vaccination patterns across the country. Importantly, major deficits in the knowledge, attitude, and practices regarding vaccination against influenza have been observed in the healthcare providers $[16,38]$ who are supposed to be the prescribers of vaccination to pregnant females. Maternal immunization during the period of influenza virus circulation has been associated with statistically significant reductions in febrile respiratory illness among mothers and infants, higher mean birth weight in infants, and lower proportion of infants who were of small-for-gestation age [39], without any attributable adverse fetal, perinatal, or infant outcomes [40]. Immunologic responses generated by influenza immunization are comparable in pregnant and nonpregnant women and can provide protection to the fetus and infant by transferring specific antibodies across the placenta [41] and in breast milk [42]. However, despite ample evidence to the contrary, misperceptions regarding the safety and efficacy of influenza vaccination are common among health care providers in India and advocate effective educational interventions.

Our study likely represents an underestimate of the total number of pregnant females with influenza during the period under study and an overestimate of the proportion of pregnant women with severe illness. Influenza positive women in the early stages might not have been aware of the status of their pregnancy and might not as such have reported the same. In addition, the lack of the data regarding outcomes of pregnancy is a limitation of the current study. However, these data provide the first evidence of the burden of influenza illness in pregnancy in India and provide strong motivation for additional research, particularly on birth outcomes. The data also emphasize the need for improved awareness among healthcare professionals regarding influenza vaccination during pregnancy and early initiation of antiviral therapy when influenza infection is suspected.

\section{Disclosure}

The funding source had no role in the design or conduct of the study or in the analysis and interpretation of the resulting data. The investigators did not receive any honoraria for their role in the study.

\section{Conflict of Interests}

The authors declare that there is no conflict of interests regarding the publication of this paper. 


\section{Acknowledgments}

The authors thank the staff of the influenza laboratory, SKIMS, Srinagar for their support during the conduct of the study. This study was funded by Sanofi India Pvt. Ltd., as an investigator initiated study.

\section{References}

[1] J. W. Harris, "Influenza occurring in pregnant women," Journal of the American Medical Association, vol. 72, no. 14, pp. 978-983, 1919.

[2] D. W. Freeman and A. Barno, "Deaths from Asian influenza associated with pregnancy," American Journal of Obstetrics and Gynecology, vol. 78, pp. 1172-1175, 1959.

[3] A. M. Siston, S. A. Rasmussen, M. A. Honein et al., "Pandemic 2009 influenza A(H1N1) virus illness among pregnant women in the United States," The Journal of the American Medical Association, vol. 303, no. 15, pp. 1517-1525, 2010.

[4] L. G. Mosby, S. A. Rasmussen, and D. J. Jamieson, "2009 Pandemic influenza A (H1N1) in pregnancy: a systematic review of the literature," American Journal of Obstetrics and Gynecology, vol. 205, no. 1, pp. 10-18, 2011.

[5] MMWR, "Patients hospitalized with 2009 pandemic influenza A (H1N1)-New York City, May 2009," Morbidity and Mortality Weekly Report (MMWR), vol. 58, pp. 1436-1440, 2010.

[6] E. H. Lee, C. Wu, E. U. Lee et al., "Fatalities associated with the $2009 \mathrm{H} 1 \mathrm{~N} 1$ influenza A virus in New York city," Clinical Infectious Diseases, vol. 50, no. 11, pp. 1498-1504, 2010.

[7] J. K. Louie, M. Acosta, K. Winter et al., "Factors associated with death or hospitalization due to pandemic 2009 influenza $\mathrm{A}(\mathrm{H} 1 \mathrm{~N} 1)$ infection in California," The Journal of the American Medical Association, vol. 302, no. 17, pp. 1896-1902, 2009.

[8] A. L. Fowlkes, P. Arguin, M. S. Biggerstaff et al., "Epidemiology of 2009 pandemic influenza A (H1N1) deaths in the United States, April-July 2009," Clinical Infectious Diseases, vol. 52, supplement 1, pp. S60-S68, 2011.

[9] W. J. Meijer, A. G. van Noortwijk, H. W. Bruinse, and A. M. Wensing, "Influenza virus infection in pregnancy: a review," Acta Obstetricia et Gynecologica Scandinavica, vol. 94, no. 8, pp. 797-819, 2015.

[10] A. A. Creanga, T. F. Johnson, S. B. Graitcer et al., "Severity of 2009 pandemic influenza A (H1N1) virus infection in pregnant women," Obstetrics \& Gynecology, vol. 115, no. 4, pp. 717-726, 2010.

[11] T. V. Hartert, K. M. Neuzil, A. K. Shintani et al., "Maternal morbidity and perinatal outcomes among pregnant women with respiratory hospitalizations during influenza season," American Journal of Obstetrics and Gynecology, vol. 189, no. 6, pp. 17051712, 2003.

[12] N. Michaan, S. Amzallag, I. Laskov et al., "Maternal and neonatal outcome of pregnant women infected with H1N1 influenza virus (Swine Flu)," Journal of Maternal-Fetal and Neonatal Medicine, vol. 25, no. 2, pp. 130-132, 2012.

[13] M. Pierce, J. J. Kurinczuk, P. Spark, P. Brocklehurst, and M. Knight, "Perinatal outcomes after maternal 2009/H1N1 infection: national cohort study," British Medical Journal, vol. 342, no. 7811, Article ID d3214, 2011.

[14] P. Gunasekaran, K. Krishnasamy, K. Arunagiri et al., "Influenza A/H1N1 2009 in pregnancy-experience in Tamil Nadu," Indian Journal of Scientific Research, vol. 3, pp. 31-35, 2012.
[15] A. Pramanick, S. Rathore, J. V. Peter, M. Moorthy, and J. Lionel, "Pandemic (H1N1) 2009 virus infection during pregnancy in South India," International Journal of Gynecology and Obstetrics, vol. 113, no. 1, pp. 32-35, 2011.

[16] P. A. Koul, N. K. Bali, S. Ali et al., "Poor uptake of influenza vaccination in pregnancy in northern India," International Journal of Gynecology and Obstetrics, vol. 127, no. 3, pp. 234-237, 2014.

[17] M. S. Chadha, V. A. Potdar, S. Saha et al., "Dynamics of influenza seasonality at sub-regional levels in India and implications for vaccination timing," PLoS ONE, vol. 10, no. 5, Article ID e0124122, 2015.

[18] World Health Organization, CDC Protocol of Real-Time RTPCR for Influenza A (H1N1), World Health Organization, Geneva, Switzerland, 2009, http://www.who.int/csr/resources/ publications/swineflu/CDCrealtimeRTPCRprotocol_20090428 .pdf.

[19] A. Bhalerao-Gandhi, P. Chhabra, S. Arya, and J. M. Simmerman, "Influenza and pregnancy: a review of the literature from India," Infectious Diseases in Obstetrics and Gynecology, vol. 2015, Article ID 867587, 8 pages, 2015.

[20] R. K. Chudasama, U. V. Patel, and P. B. Verma, "Hospitalizations associated with 2009 influenza A (H1N1) and seasonal influenza in Saurashtra region, India," Journal of Infection in Developing Countries, vol. 4, no. 12, pp. 834-841, 2010.

[21] A. A. Mehta, V. A. Kumar, S. G. Nair, F. K. Joseph, G. Kumar, and S. K. Singh, "Clinical profile of patients admitted with swine-origin influenza A (H1N1) virus infection: an experience from a tertiary care hospital," Journal of Clinical and Diagnostic Research, vol. 7, no. 10, pp. 2227-2230, 2013.

[22] V. Sharma, P. K. Verma, S. Gupta, and A. Sharma, "Mortality from influenza A/H1N1 in a tertiary care teaching institution in North India," Journal of Infection in Developing Countries, vol. 4, no. 8, pp. 468-471, 2010.

[23] K. Ramakrishna, S. Sampath, J. Chacko et al., "Clinical profile and predictors of mortality of severe pandemic (H1N1) 2009 virus infection needing intensive care: a multi-centre prospective study from South India," Journal of Global Infectious Diseases, vol. 4, no. 3, pp. 145-152, 2012.

[24] K. M. Neuzil, G. W. Reed, E. F. Mitchel, L. Simonsen, and M. R. Griffin, "Impact of influenza on acute cardiopulmonary hospitalizations in pregnant women," American Journal of Epidemiology, vol. 148, no. 11, pp. 1094-1102, 1998.

[25] V. L. Rogers, J. S. Sheffield, S. W. Roberts et al., "Presentation of seasonal influenza A in pregnancy: 2003-2004 influenza season," Obstetrics and Gynecology, vol. 115, no. 5, pp. 924-929, 2010.

[26] D. L. Schanzer, T. W. S. Tam, J. M. Langley, and B. T. Winchester, “Influenza-attributable deaths, Canada 1990-1999," Epidemiology and Infection, vol. 135, no. 7, pp. 1109-1116, 2007.

[27] L. Dodds, S. A. McNeil, D. B. Fell et al., "Impact of influenza exposure on rates of hospital admissions and physician visits because of respiratory illness among pregnant women," Canadian Medical Association Journal, vol. 176, no. 4, pp. 463-468, 2007.

[28] D. J. Jamieson, M. A. Honein, S. A. Rasmussen et al., "H1N1 2009 influenza virus infection during pregnancy in the USA," The Lancet, vol. 374, no. 9688, pp. 451-458, 2009.

[29] S. Mathur, T. Dubey, M. Kulshrestha et al., "Clinical profile and mortality among novel influenza A (H1N1) infected patients: 2009-2010 Jodhpur, Rajasthan pandemic," Journal of Association of Physicians of India, vol. 61, no. 9, pp. 627-632, 2013. 
[30] G. Dubar, E. Azria, A. Tesnière et al., "French experience of 2009 A/H1N1v influenza in pregnant women," PLoS ONE, vol. 5, no. 10, Article ID e13112, 2010.

[31] E. Maraví-Poma, I. Martin-Loeches, E. Regidor et al., "Severe 2009 A/H1N1v influenza in pregnant women in Spain," Critical Care Medicine, vol. 39, no. 5, pp. 945-951, 2011.

[32] H. E. Mighty, "Acute respiratory failure in pregnancy," Clinical Obstetrics and Gynecology, vol. 53, no. 2, pp. 360-368, 2010.

[33] CDC, Pregnant Women and Influenza, CDC, 2015, http://www .cdc.gov/flu/protect/vaccine/pregnant.htm.

[34] A. P. Kourtis, J. S. Read, and D. J. Jamieson, "Pregnancy and infection," The New England Journal of Medicine, vol. 370, no. 23, pp. 2211-2218, 2014.

[35] T. A. Kraus, R. S. Sperling, S. M. Engel et al., "Peripheral blood cytokine profiling during pregnancy and post-partum periods," American Journal of Reproductive Immunology, vol. 64, no. 6, pp. 411-426, 2010.

[36] C. B. Bridges, K. Fukuda, N. J. Cox, and J. A. Singleton, "Prevention and control of influenza. Recommendations of the Advisory Committee on Immunization Practices (ACIP)," MMWR Recommendations and Reports, vol. 50, no. 4, pp. 1-44, 2001.

[37] FOGSI, Vaccination in women, 2014, http://www.fogsi.org/wpcontent/uploads/2015/11/vaccination_women.pdf.

[38] N. K. Bali, U. H. Khan, F. Ahmad, M. A. Ashraf, R. B. Lal, and P. A. Koul, "Knowledge, attitude, and practices about the seasonal influenza vaccination among healthcare workers in Srinagar, India," Influenza and Other Respiratory Viruses, vol. 7, no. 4, pp. 540-545, 2012.

[39] M. C. Steinhoff, S. B. Omer, E. Roy et al., "Neonatal outcomes after influenza immunization during pregnancy: a randomized controlled trial," Canadian Medical Association Journal, vol. 184, no. 6, pp. 645-653, 2012.

[40] K. Zaman, E. Roy, S. E. Arifeen et al., "Effectiveness of maternal influenza immunization in mothers and infants," The New England Journal of Medicine, vol. 359, no. 15, pp. 1555-1564, 2008.

[41] J. M. Puck, W. P. Glezen, A. L. Frank, and H. R. Six, "Protection of infants from infection with influenza A virus by transplacentally acquired antibody," Journal of Infectious Diseases, vol. 142, no. 6, pp. 844-849, 1980.

[42] E. P. Schlaudecker, M. C. Steinhoff, S. B. Omer et al., "IgA and neutralizing antibodies to influenza A virus in human milk: a randomized trial of antenatal influenza immunization," PLoS ONE, vol. 8, no. 8, Article ID e70867, 2013. 


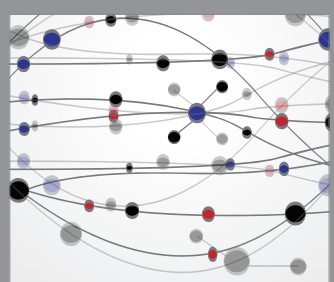

The Scientific World Journal
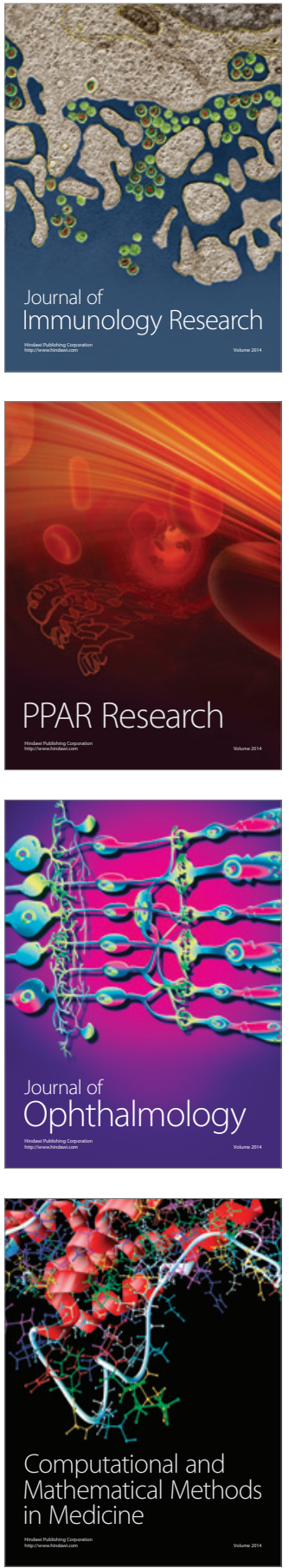

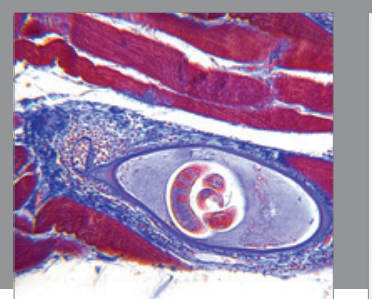

Gastroenterology Research and Practice

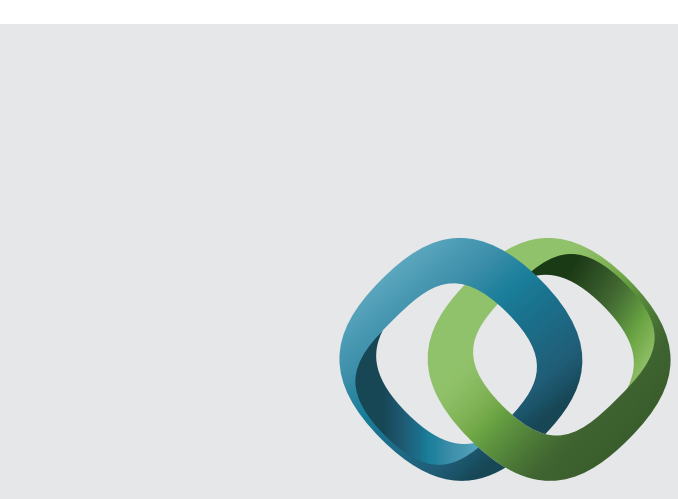

\section{Hindawi}

Submit your manuscripts at

http://www.hindawi.com
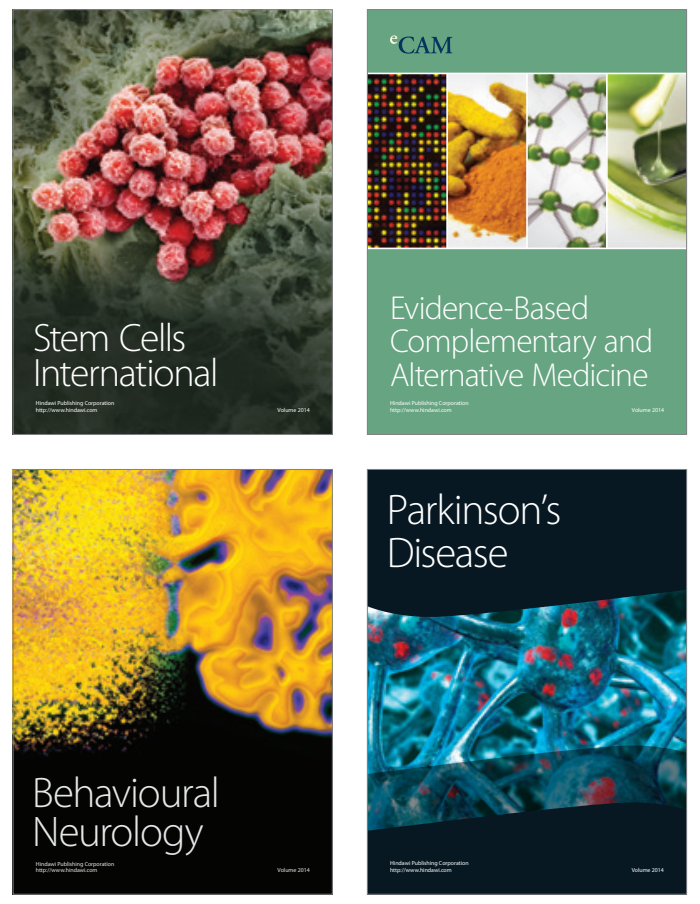
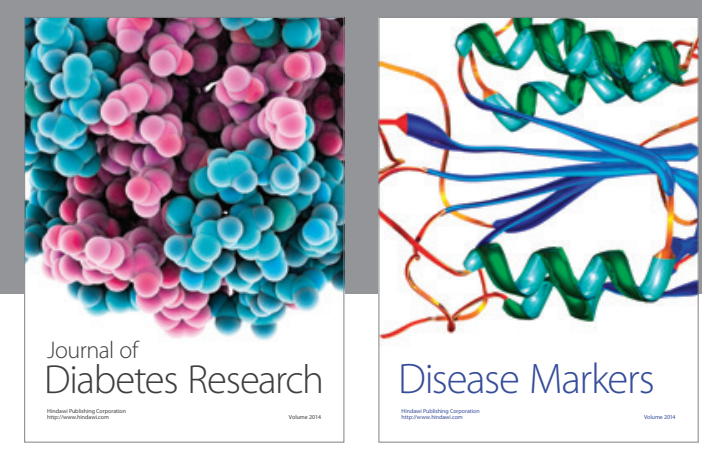

Disease Markers
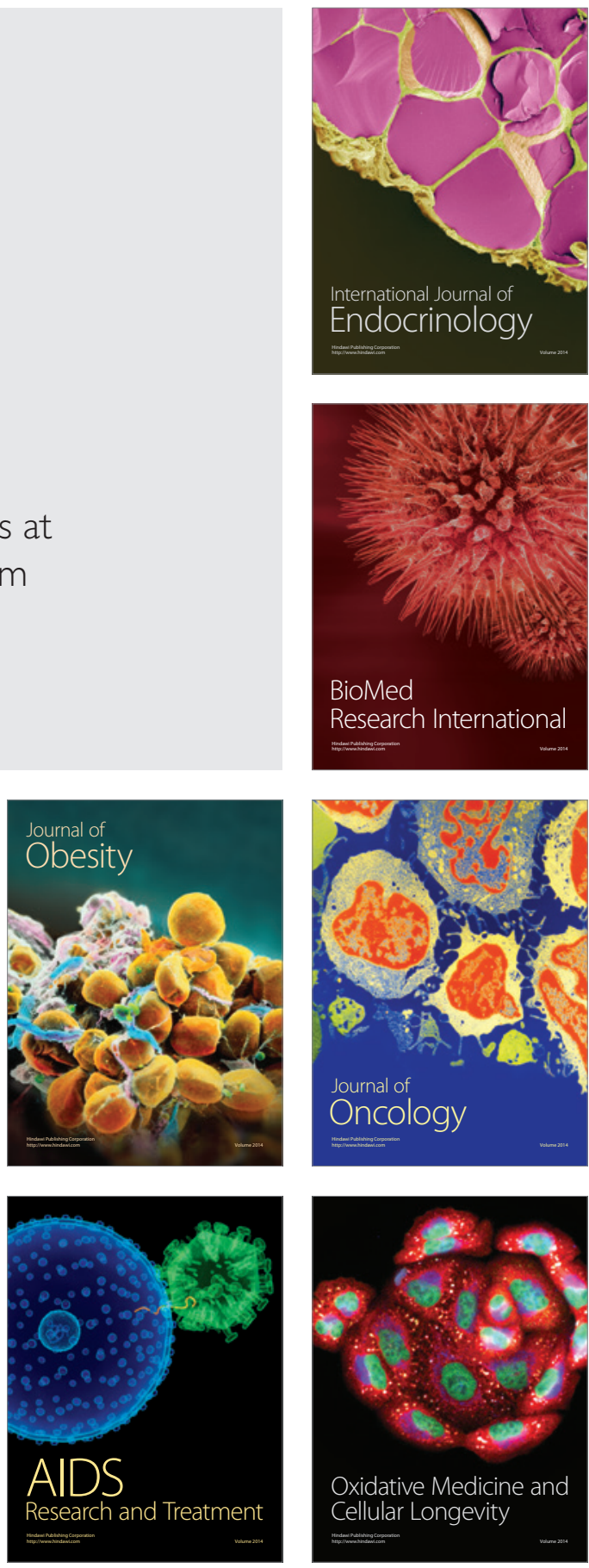